\title{
Probing the Influence of Substrate Hole Shape on the Interaction between Helium Ion and Suspended Monolayer Graphene with Raman Spectroscopy
}

Shixuan He ${ }^{1,2 \#}$, Wanyi Xie $e^{1,3 \#, ~ Y o n g n a ~ Z h a n g ~}{ }^{1}$, Shaoxi Fang ${ }^{1,3}$, Daming Zhou ${ }^{1}$, Jie

Gan $^{1}$, Zhiyou Zhang ${ }^{2}$, Jinglei Du ${ }^{2}$, Chunlei Du ${ }^{2 *}$ \& Deqiang Wang ${ }^{1,3^{*}}$

${ }^{1}$ Chongqing Institute of Green and Intelligent Technology, Chinese Academy of Sciences, Chongqing, 400714, P. R. China

${ }^{2}$ College of Physics, Sichuan University, Chengdu, Sichuan, 610065, P. R. China

${ }^{3}$ Chongqing School, University of Chinese Academy of Sciences, Chongqing, 400714, P. R. China

*E-mail address: mscldu@cigit.ac.cn,dqwang@cigit.ac.cn 


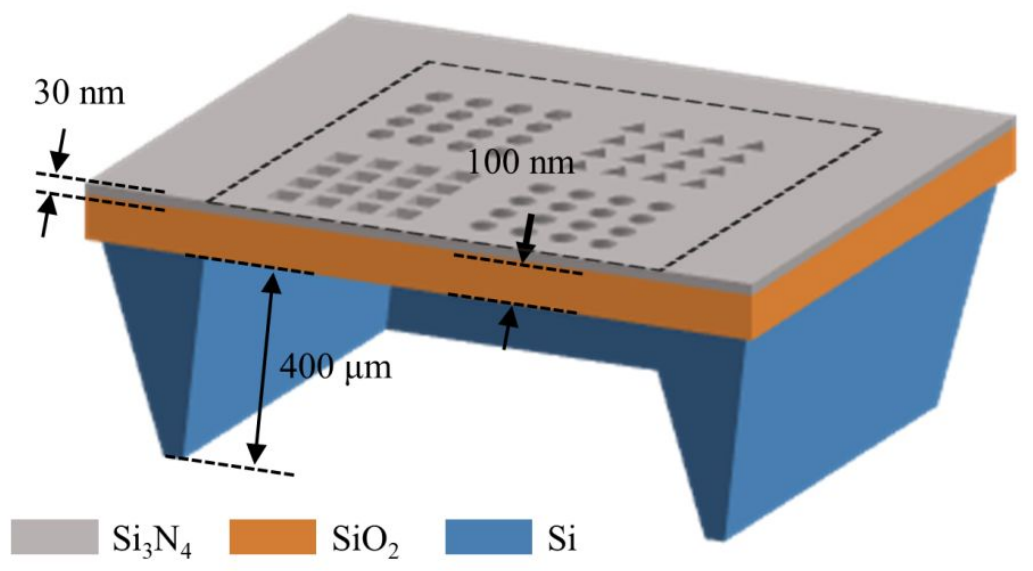

Figure S1. The schematic of the $\mathrm{Si}_{3} \mathrm{~N}_{4} / \mathrm{SiO}_{2}$ substrate with pore structures of different shapes fabricated by Ga-FIB technology.

(a)
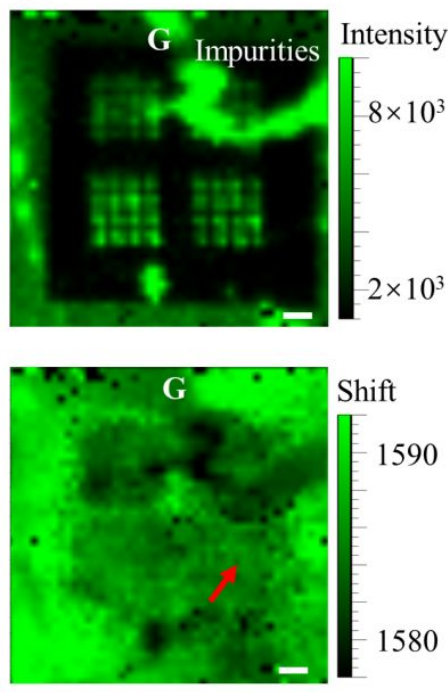
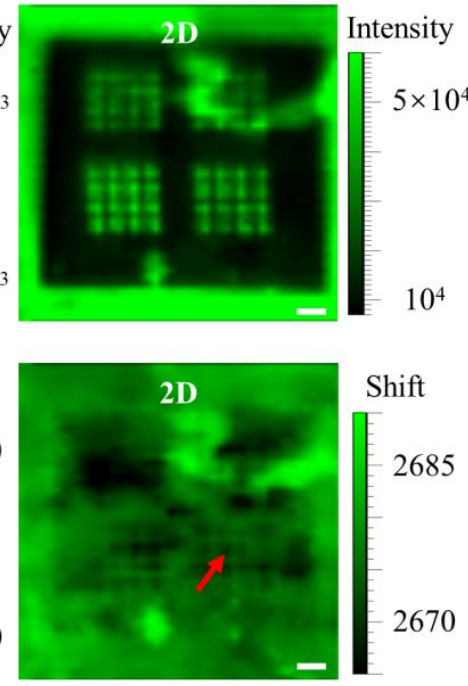

(b)

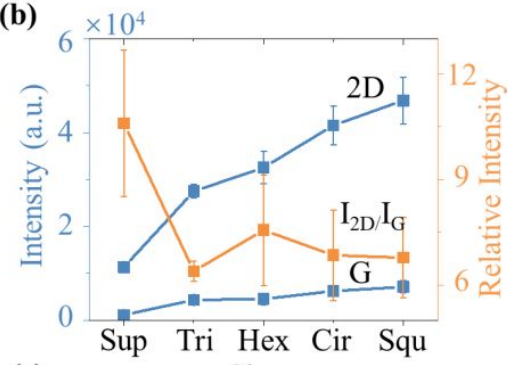

(c)

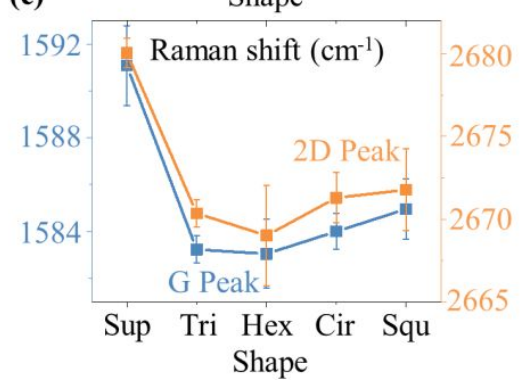

Figure S2. Raman mapping of suspended graphene on a substrate with the periodic pores of different shapes and its Raman characteristics. (a) Raman mapping (scanning step: $0.5 \mu \mathrm{m}$ ) of the suspended graphene by using the $532 \mathrm{~nm}$ wavenumber laser, including its intensities (a.u.) and Raman shifts $\left(\mathrm{cm}^{-1}\right)$ of $\mathrm{G}$ and 2D peaks, there were $10-20 \mathrm{~cm}^{-1}$ redshifts at the $\mathrm{G}$ and $2 \mathrm{D}$ peaks for the suspended graphene in comparing with that of graphene over $\mathrm{Si}_{3} \mathrm{~N}_{4} / \mathrm{SiO}_{2}$ substrate respectively (red arrows, scale bar: 2 $\mu \mathrm{m}$ ). (b) Raman intensities of graphene $\mathrm{G}$ and 2D peaks, and their relative intensities 
for four shape pores substrate (Sup: Supported). (c) Raman shifts of graphene G and 2D peaks for four shape pores substrate in comparing with that of graphene over $\mathrm{Si}_{3} \mathrm{~N}_{4} / \mathrm{SiO}_{2}$ substrate. Error bars represent standard error of the mean (s. e. m.) $(\mathrm{n}=16$, except for triangle shape substrate $n=4$ ).

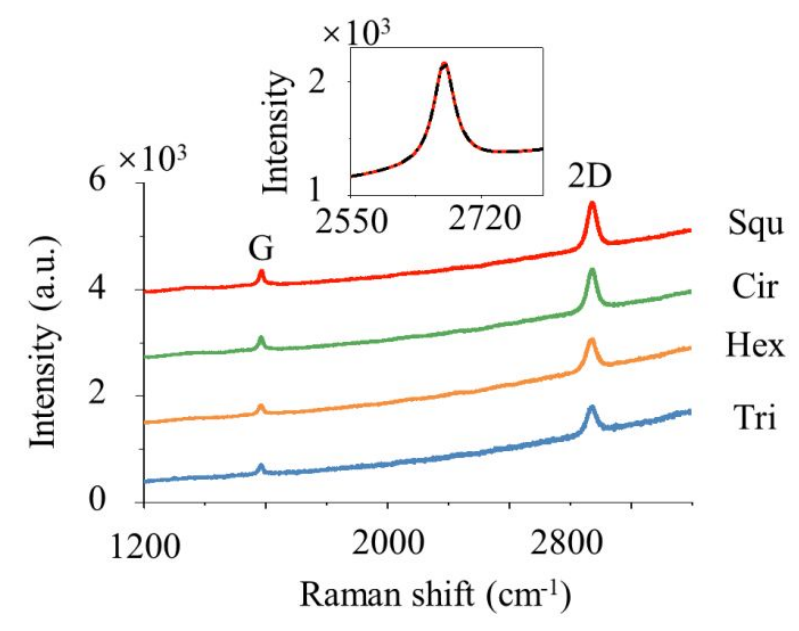

Figure S3. The average spectra of the suspended graphene on the different shape pores. The inset Figure shows the fitting result for the graphene 2D peak, it was well fitted with a Lorentz function. 
(a)

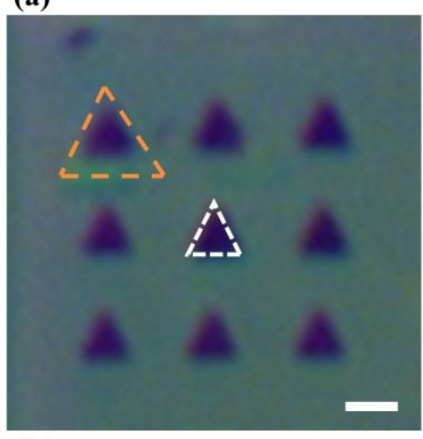

(b)

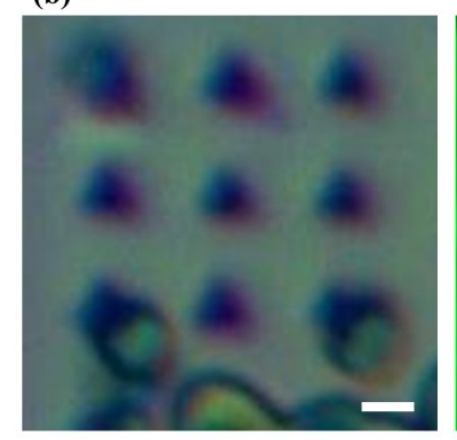

(c) Scanning step: $0.5 \mu \mathrm{m}$
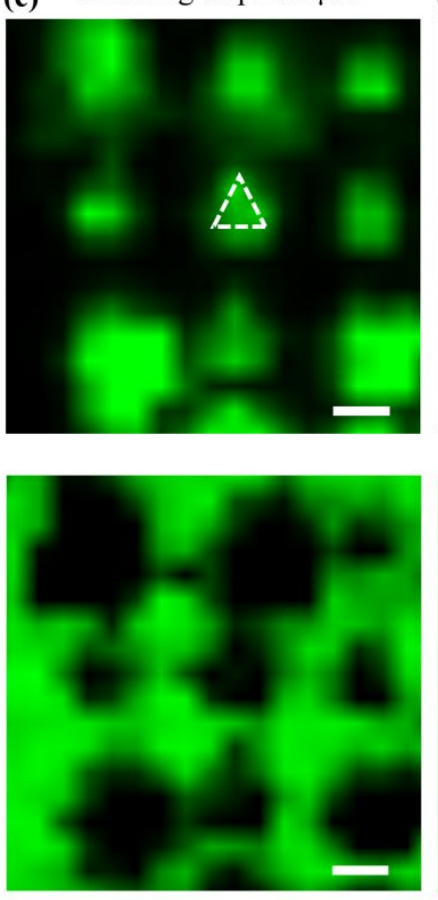

(d) Scanning step: $0.3 \mu \mathrm{m}$
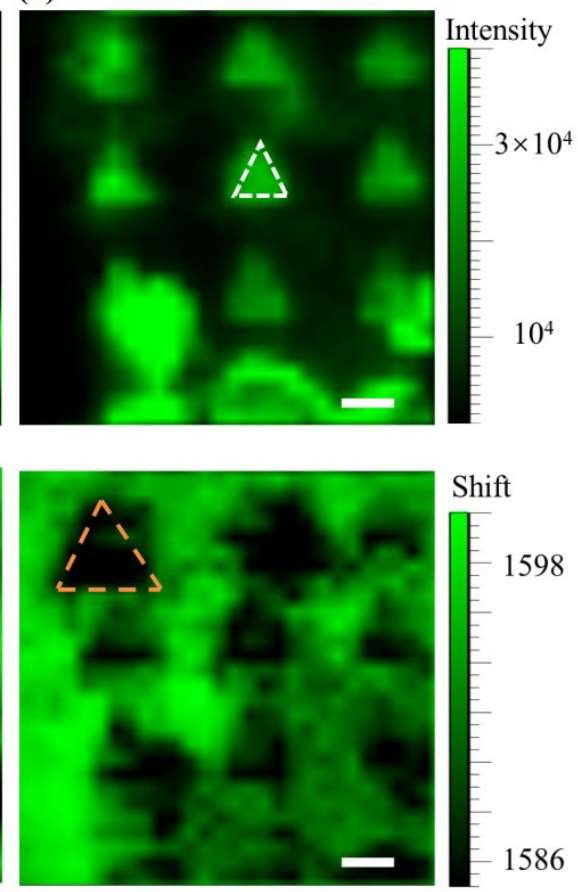

Figure S4. The suspended monolayer graphene on the triangle holes substrate and its

Raman characteristics $(\mathrm{D}=1 \mu \mathrm{m}$, scale bar: $1 \mu \mathrm{m})$. (a) The optical microscope of the triangle holes structures on the $\mathrm{Si}_{3} \mathrm{~N}_{4} / \mathrm{SiO}_{2}$ substrate and (b) the suspended monolayer graphene over the triangle holes substrate. Raman mapping of suspended monolayer graphene by using $532 \mathrm{~nm}$ wavenumber laser with a scanning step of $0.5 \mu \mathrm{m}$ (c) and $0.3 \mu \mathrm{m}(\mathrm{d})$ respectively, including its intensities (a.u.) and Raman shifts $\left(\mathrm{cm}^{-1}\right)$ at $\mathrm{G}$ peak, there were $10-20 \mathrm{~cm}^{-1}$ redshifts at the $\mathrm{G}$ peak for the suspended graphene. Raman mapping of suspended graphene at $G$ peak clearly shows a triangular shape similar to the optical microscope image (white and orange dotted triangle). 
(a)

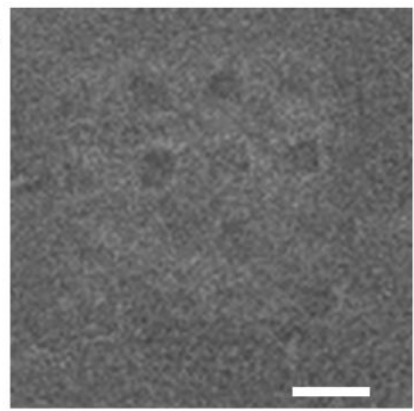

(b)

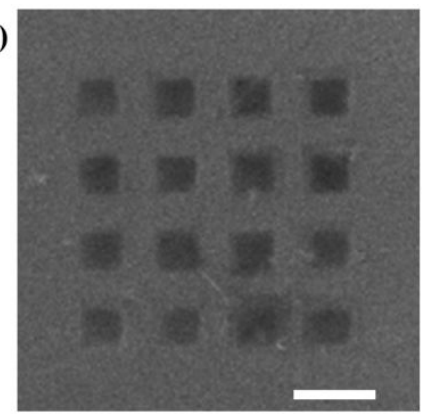

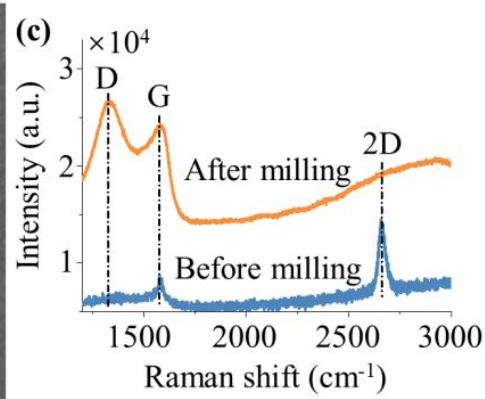

Figure S5. The suspended monolayer graphene on the substrate with square holes and its Raman characteristics. HIM images of suspended graphene (a) before (beam current: $1.29 \mathrm{pA}$ ) and (b) after (beam current: $1.62 \mathrm{pA}$ ) milling by using HIM with high HIB current (scale bar: $1 \mu \mathrm{m}$ ). (c) Raman characteristics of suspended graphene before and after HIB milling.

Table S1. The HIB doses used for the substrate with pore structures of different shapes (Dose: ions)

\begin{tabular}{cccccccccc}
\hline & Substrate & 1 & 2 & 3 & 4 & 5 & 6 & 7 & 8 \\
\hline \multirow{5}{*}{ Doses } & Square holes $\left(\times 10^{6}\right)$ & 2.26 & 1.3 & 1.97 & 1.2 & 0.13 & 0.13 & 0.84 & 0.18 \\
& Triangle holes $\left(\times 10^{4}\right)$ & 2.79 & 3.02 & 3.02 & 0.88 & 8.9 & 7.48 & 6.52 & 7.48 \\
& Circle holes $\left(\times 10^{5}\right)$ & 13 & 6.5 & 3 & 0.94 & 0.02 & 0.03 & 0.07 & 0.08 \\
& Hexagon holes $\left(\times 10^{6}\right)$ & 2.74 & 3.02 & 1.97 & 2.54 & 0.75 & 1.03 & - & - \\
\hline \multirow{5}{*}{ Doses } & Substrate & 9 & 10 & 11 & 12 & 13 & 14 & 15 & 16 \\
\hline & Square holes $\left(\times 10^{4}\right)$ & 3.67 & 2.24 & 5.1 & 4.62 & 0.88 & 3.74 & 3.26 & 3.98 \\
& Triangle holes $\left(\times 10^{5}\right)$ & 5.1 & 5.57 & 7 & 7.96 & 0 & 0 & 0 & 0 \\
& Circle holes $\left(\times 10^{5}\right)$ & 0 & 0 & 0 & 0 & 0 & 0 & 0 & 0 \\
& Hexagon holes $\left(\times 10^{5}\right)$ & 5.17 & 3.63 & - & - & 0.79 & - & - & - \\
\hline
\end{tabular}



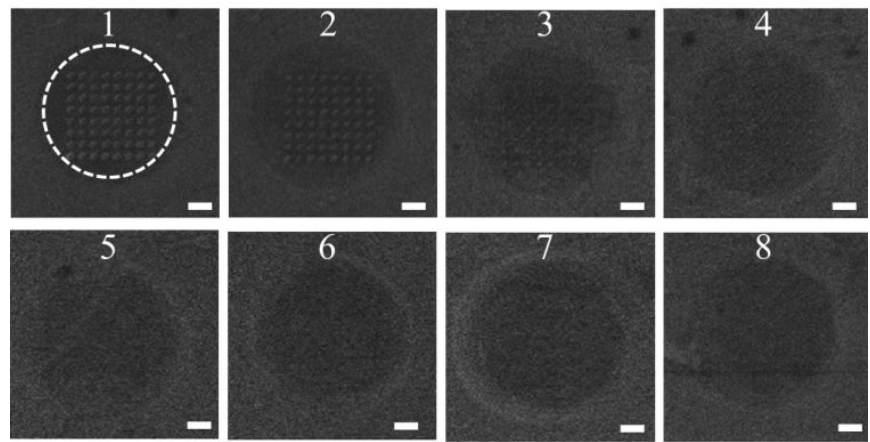

Figure S6. HIM images (beam current: $\sim 0.2 \mathrm{pA}$; scale bar: $100 \mathrm{~nm}$ ) of suspended graphene on a substrate with circle holes after HIB milling with different doses.

(a)

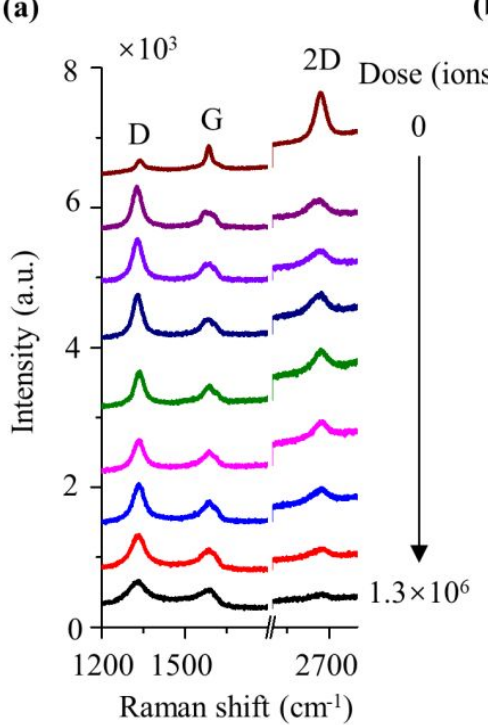

(b)
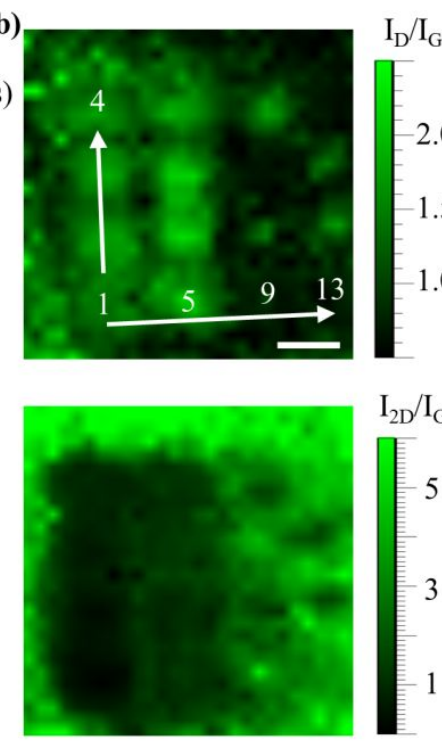

(c)

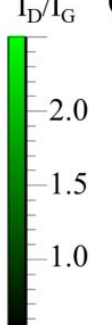

12

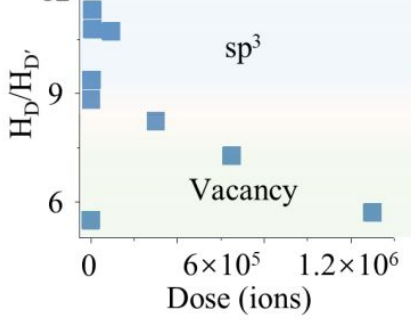

(d)

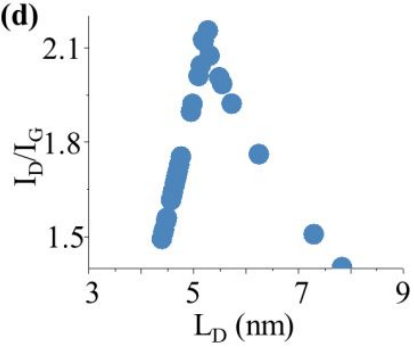

Figure S7. (a) The average Raman spectra of suspended graphene on circle holes substrate after HIB milling with different doses. (b) Raman mapping characteristic relative intensities $\mathrm{I}_{\mathrm{D}} / \mathrm{I}_{\mathrm{G}}$ and $\mathrm{I}_{2 \mathrm{D}} / \mathrm{I}_{\mathrm{G}}$ of suspended graphene on circle shape after HIB milling with different doses. (c) Relative heights $\mathrm{H}_{\mathrm{D}} / \mathrm{H}_{\mathrm{D}^{\prime}}$ of suspended graphene on circle holes substrate after HIB milling with different doses. (d) The calculated average distance between defects $\mathrm{L}_{\mathrm{D}}$ correlate with relative intensities $\mathrm{I}_{\mathrm{D}} / \mathrm{I}_{\mathrm{G}}$. 

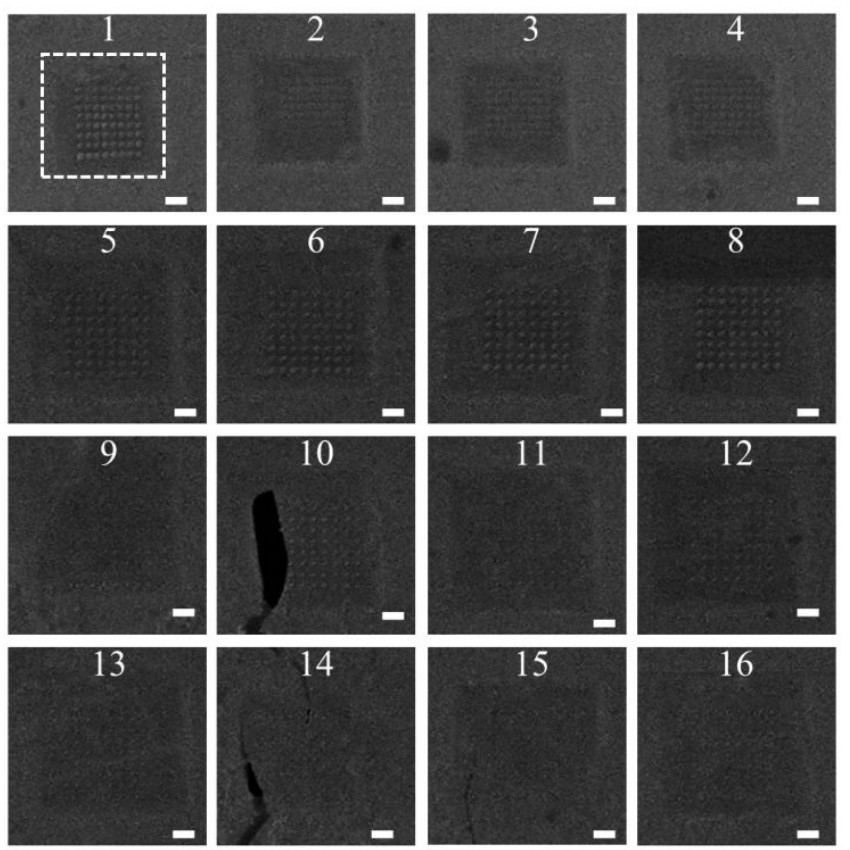

Figure S8. HIM images (beam current: $\sim 0.2 \mathrm{pA}$; scale bar: $100 \mathrm{~nm}$ ) of suspended graphene on a substrate with square holes after HIB milling with different doses.

(a)
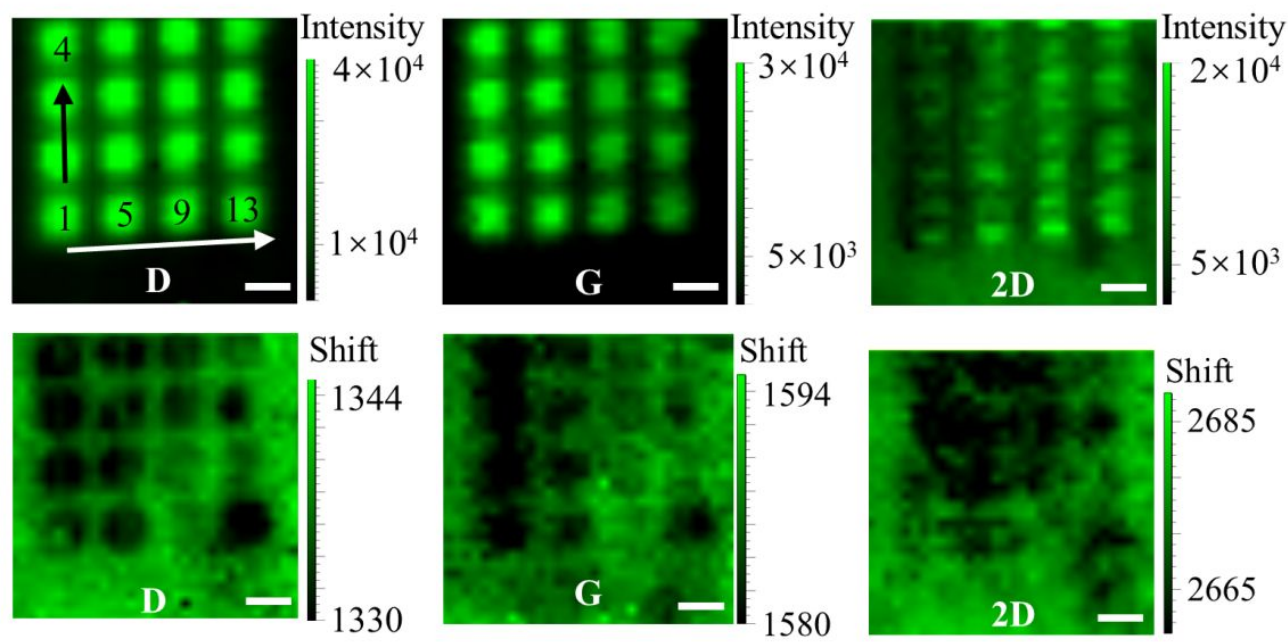

(b)

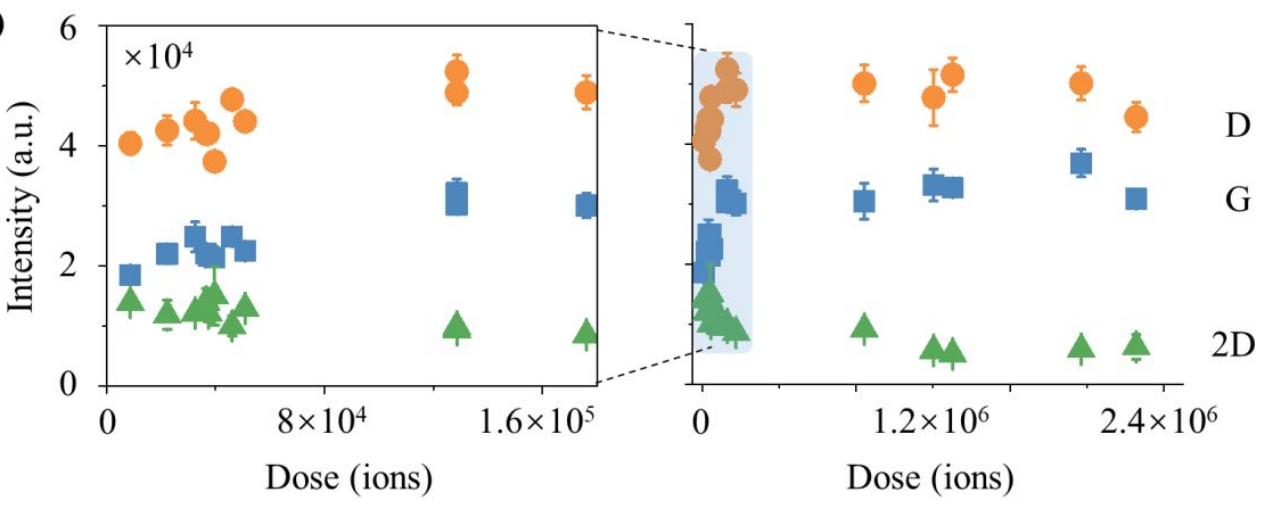

Figure S9. Raman characteristics of engineered defects in suspended monolayer 
graphene on the substrate with square holes. (a) Raman mapping (scanning step: 0.2 $\mu \mathrm{m})$ of suspended graphene on square shape after HIB milling with different doses, including its intensities and shifts of D, G and 2D Peaks (scale bar: $1 \mu \mathrm{m}$ ). (b) Raman characteristics of suspended graphene along with different HIB doses, the left part of (b) is enlarged of Raman characteristics of suspended graphene along with low HIB doses. Error bars represent s. e. m. $(n=4)$.

(a)
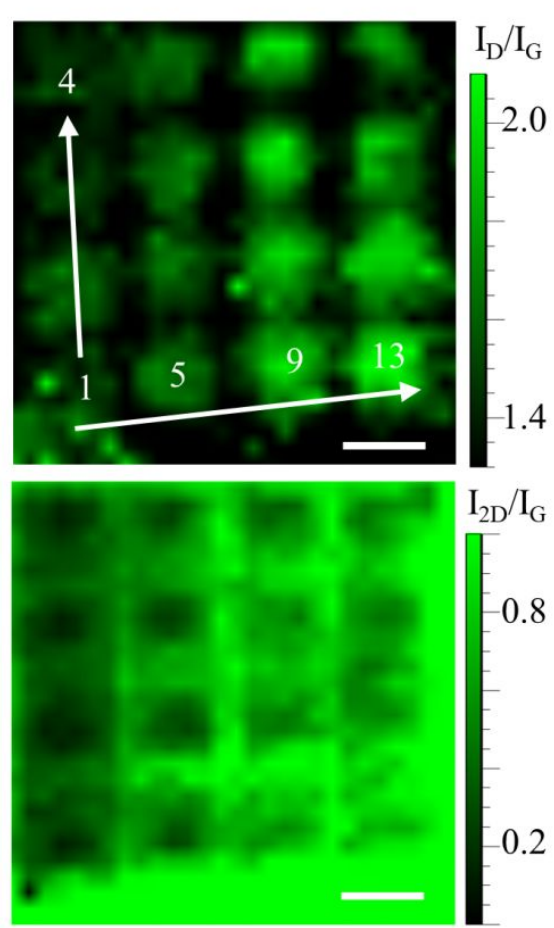

(c) (b)
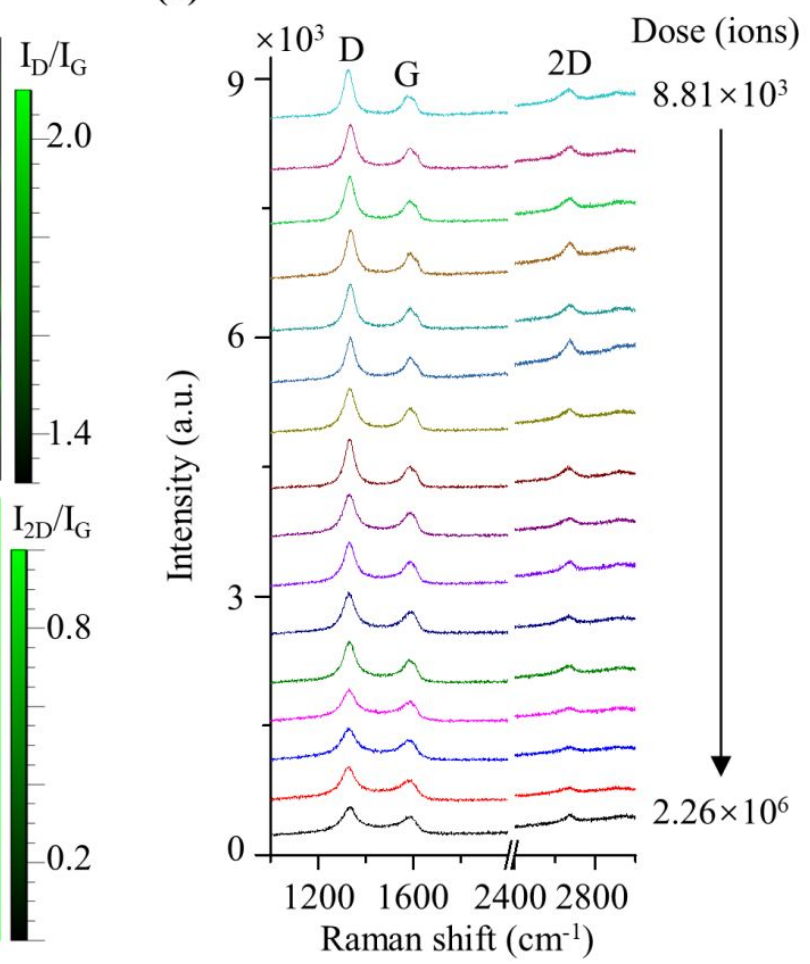
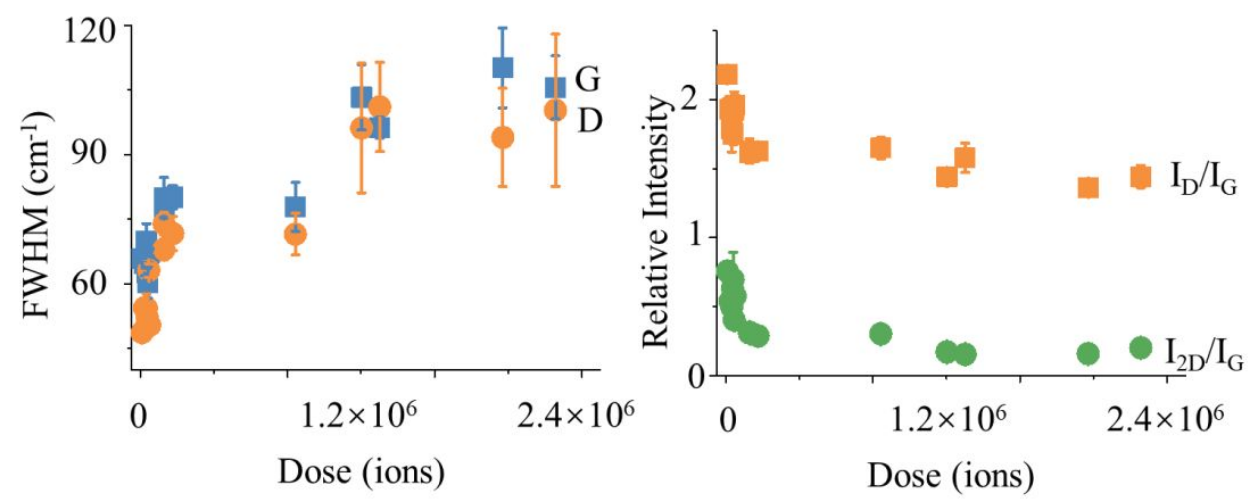
Figure S10. Raman characteristics of engineered defects in suspended monolayer graphene on the substrate with square holes. (a) Raman mapping (scanning step: 0.2 $\mu \mathrm{m})$ of suspended graphene on square shape after HIB milling with different doses, including its intensities and shifts of $\mathrm{D}, \mathrm{G}$ and 2D Peaks (scale bar: $1 \mu \mathrm{m}$ ). (b) Raman characteristics of suspended graphene along with different HIB doses, the left part of (b) is enlarged of Raman characteristics of suspended graphene along with low HIB doses. Error bars represent s. e. m. $(n=4)$.

(a)
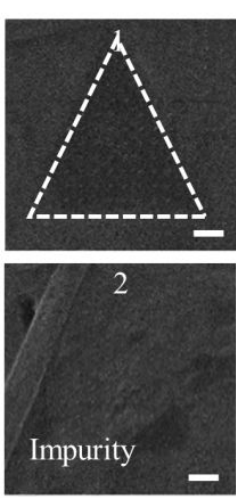

3
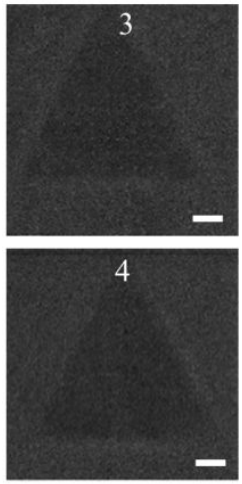
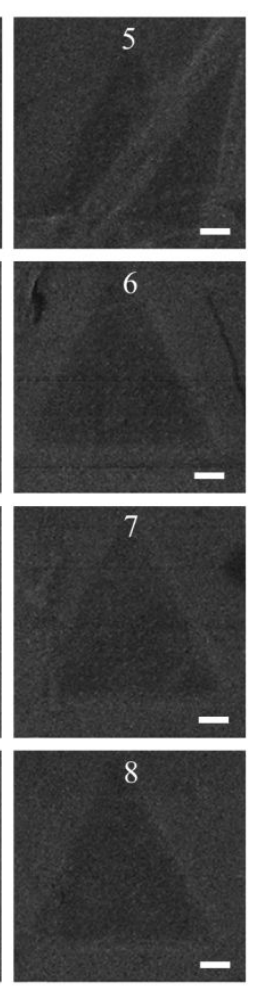
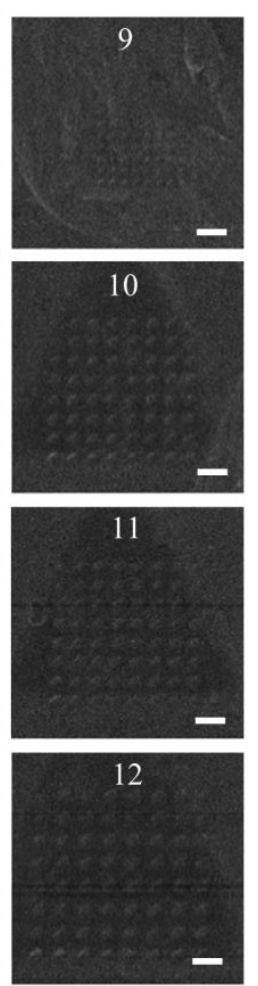

(b)

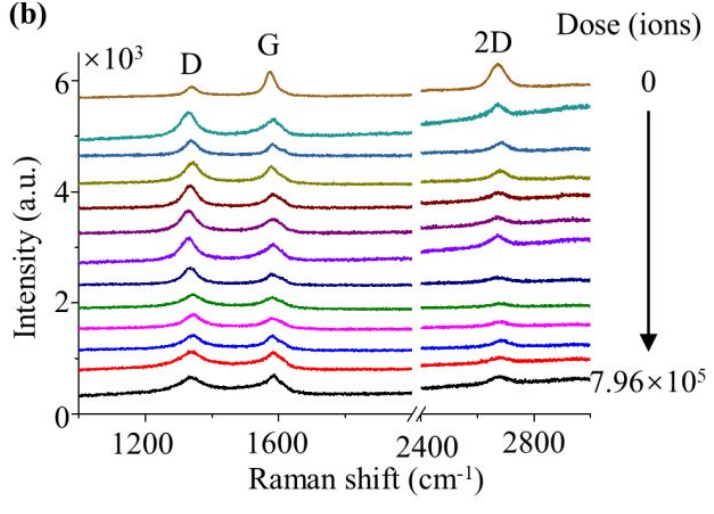

(c)

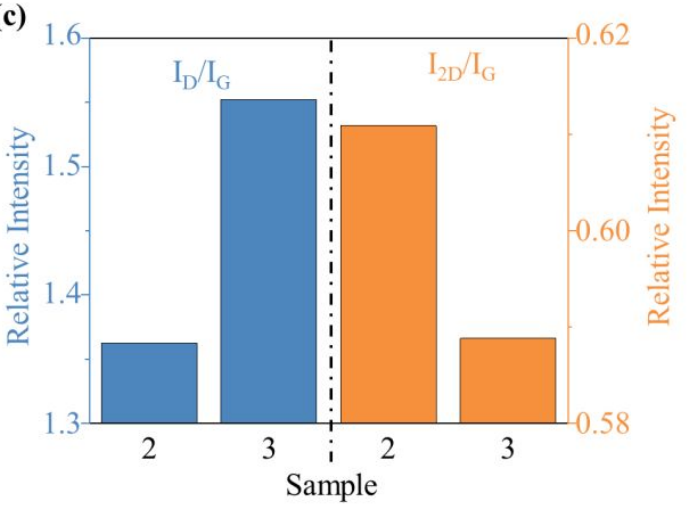

Figure S11 HIM images of suspended graphene on a substrate with triangle holes and its average Raman spectra. (a) HIM images (beam current: $\sim 0.2 \mathrm{pA}$; scale bar: $100 \mathrm{~nm}$ ) and (b) the average Raman spectra of suspended graphene on triangle holes substrate after HIB milling with different doses. (c) The relative intensities of suspended 
graphene on triangle holes substrate (samples 2 and 3) after HIB milling with the same dose $\left(3.02 \times 10^{4}\right)$.
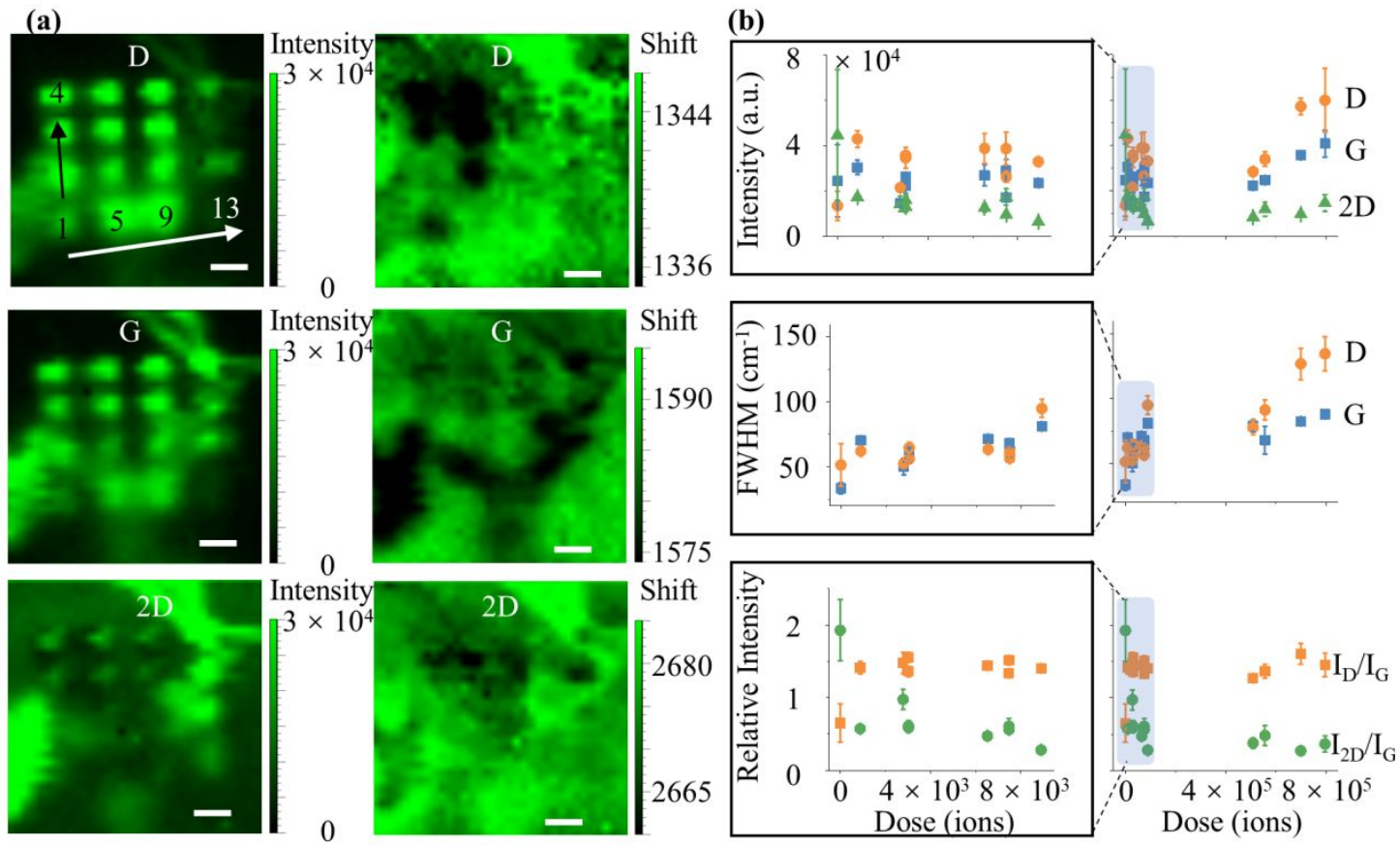

Figure S12. Raman characteristics of engineered defects in suspended monolayer graphene on the substrate with triangle holes. (a) Raman mapping (scanning step: 0.2 $\mu \mathrm{m})$ of suspended graphene on triangle shape after HIB milling with different doses, including its intensities and shifts of $\mathrm{D}, \mathrm{G}$ and 2D peaks (scale bar: $1 \mu \mathrm{m}$ ). (b) Raman characteristics of the suspended graphene along with different HIB doses, the left part of (b) is enlarged of Raman characteristics of the suspended graphene along with low HIB doses. Error bars represent s. e. m. $(n=4)$. 

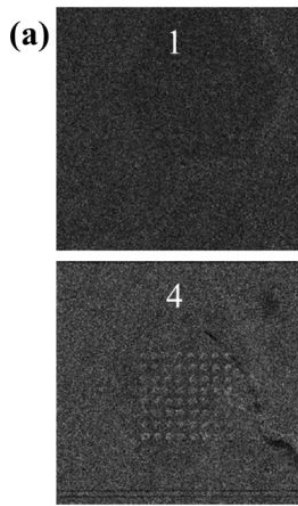

9
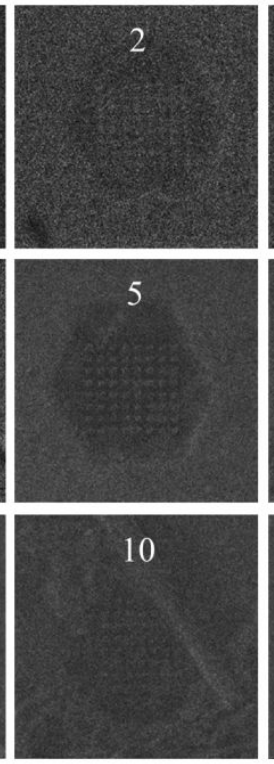
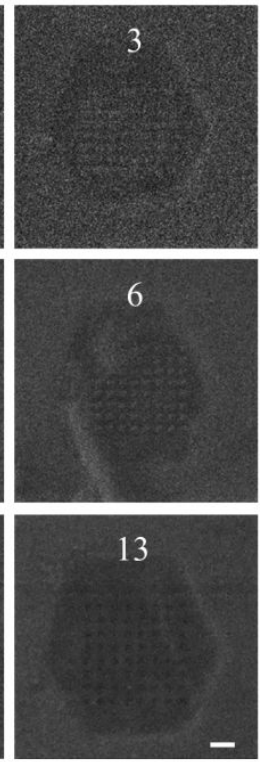

(b)

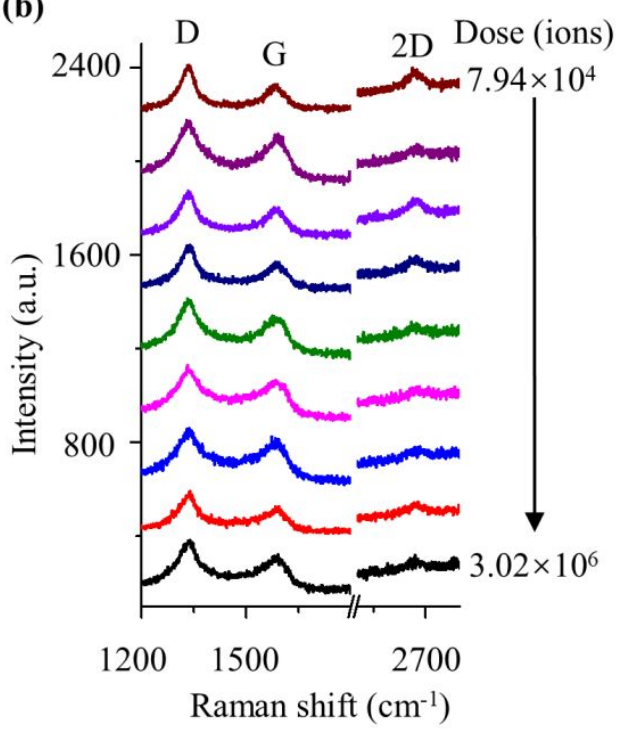

Figure S13. HIM images and average spectra of suspended graphene on the substrate with the hexagon holes after HIB milling with different doses. (a) HIM images of suspended graphene on the substrate with the hexagon holes (beam current: $\sim 0.21 \mathrm{pA}$; scale bar: $100 \mathrm{~nm}$ ) and (b) its average spectra.
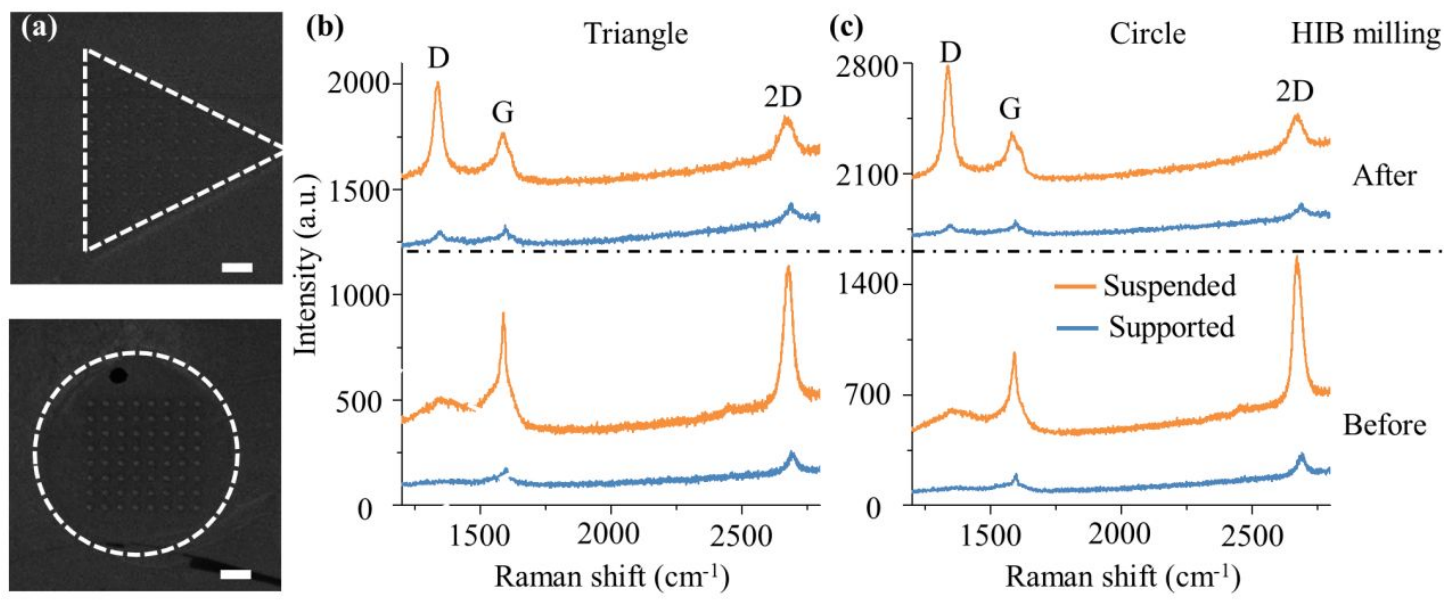

Figure S14. HIM images and average spectra of monolayer graphene on the substrate with the circle and triangle holes $(\mathrm{D}=1 \mu \mathrm{m})$. (a) HIM images of monolayer graphene on the substrate with the triangle and circle holes after HIB milling with $6.24 \times 10^{4}$ and $2.71 \times 10^{5}$ ions dosages respectively (beam current: $\sim 0.15 \mathrm{pA}$; scale bar: $200 \mathrm{~nm}$ ) and 
(b and $c)$ its average spectra before and after HIB milling.

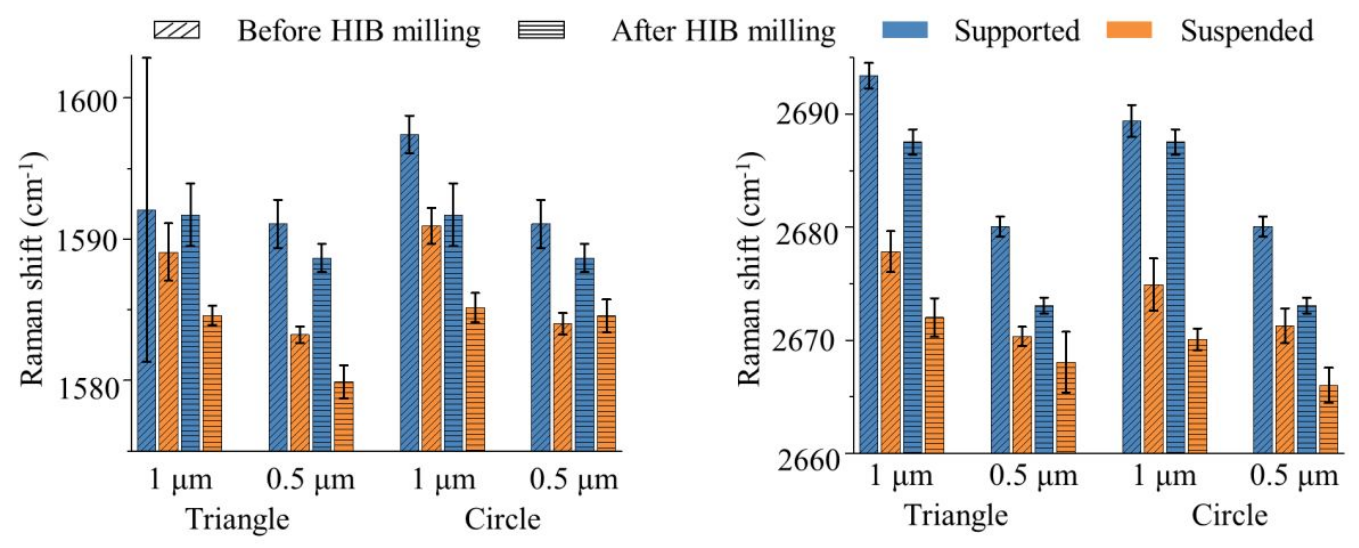

Figure S15. Raman characteristics shifts of suspended and supported monolayer graphene before and after HIB milling with different freestanding areas. Error bars represent s. e. m. $(n=4)$.

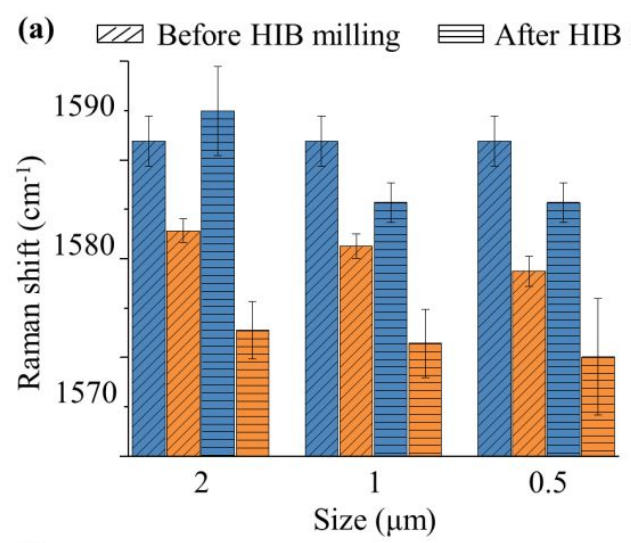

(c)

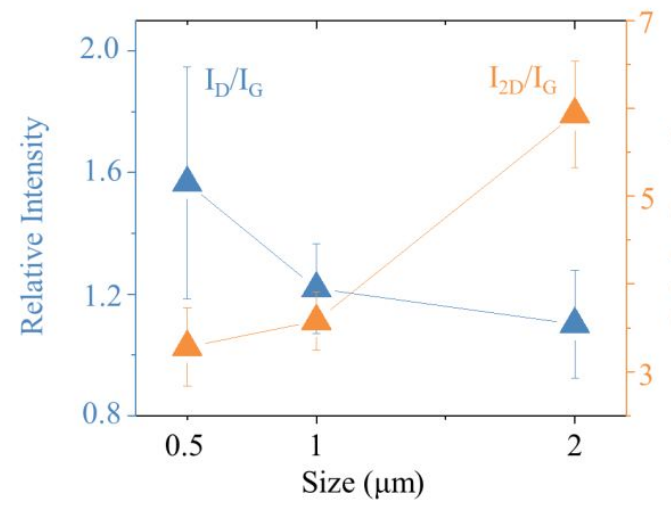

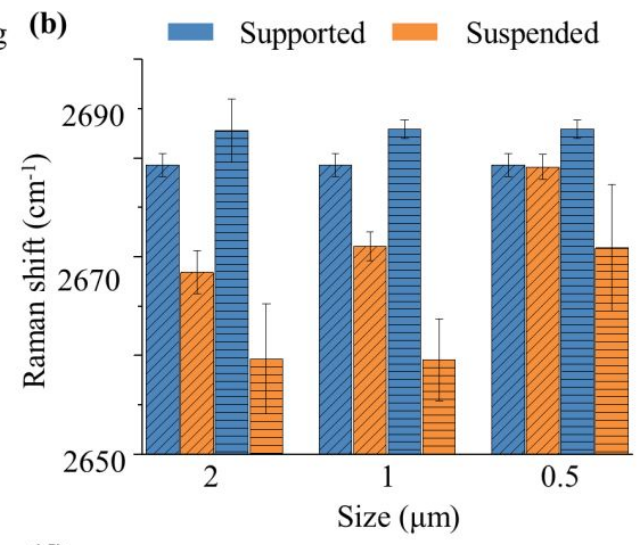

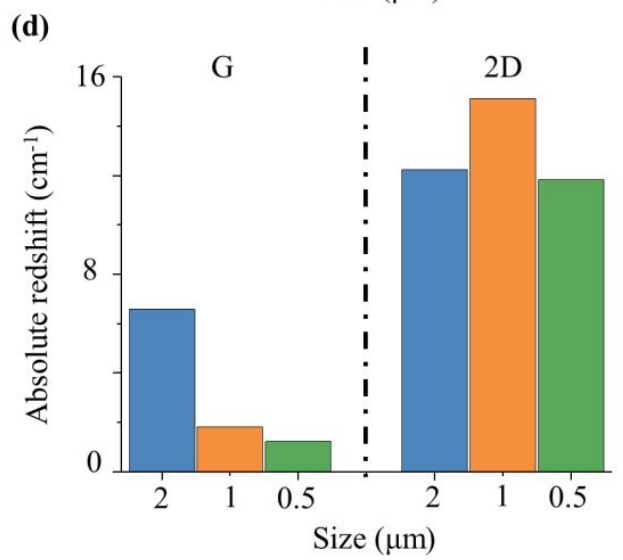

Figure S16. Substrate pore size effect of defects engineered in suspended monolayer graphene upon helium ions bombardment. Raman characteristics shifts at (a) G and (b) 
2D peaks of suspended and supported monolayer graphene before and after HIB milling with different sizes. The relative intensities (c) and absolute redshifts (d) of suspended monolayer graphene on holes of different sizes substrate after helium ions bombardment. Error bars represent s. e. m. $(n=4)$.

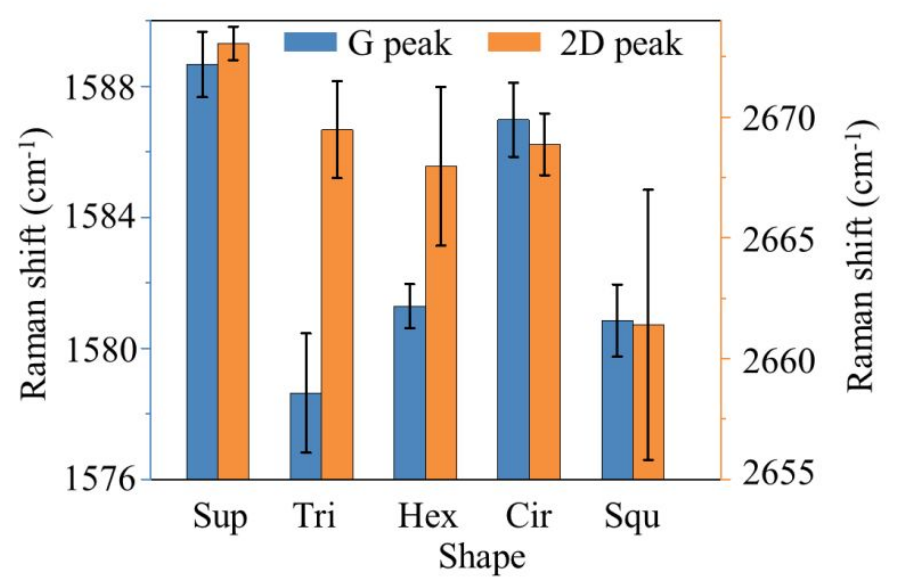

Figure S17. Raman characteristics shifts ( $G$ and 2D peaks) of monolayer graphene for four shape pores substrate in comparing with that of graphene over $\mathrm{Si}_{3} \mathrm{~N}_{4} / \mathrm{SiO}_{2}$ substrate after HIB milling. Error bars represent s. e. m. $(n=4)$. 